\title{
Curcumin protects against rotenone-induced neurotoxicity in cell and drosophila models of Parkinson's disease ${ }^{*}$
}

\author{
Zhaohui Liü, Tianxia Li, Dejun Yang, Wanli W. Smith ${ }^{\#}$ \\ Department of Pharmaceutical Sciences, University of Maryland School of Pharmacy, Baltimore, USA; \\ \#Corresponding Authors: liuzhaohui1105@hotmail.com,wsmith@,rx.umaryland.edu
}

Received 27 November 2012; revised 8 January 2013; accepted 16 January 2013

\begin{abstract}
Parkinson's disease (PD) is a progressive neurodegenerative movement disorder resulting from a selective loss of dopaminergic neurons. The pathogenesis of PD remains incompletely understood, but increasing evidence from human and animal studies has suggested that oxidative damage contributes to the neuronal loss in PD. In this study, we used rotenone (a mitochondrial complex I inhibitor) based cell and Drosophila models that resemble some key pathological features of PD to test whether curcumin, a potent antioxidant compound, derived from the curry spice turmeric, could protect against rotenone-induced neuronal toxicity. We found that curcumin reduced rotenone induced cell death in SH-SY5Y human neuroblastoma cells and alleviated PD-like symptoms in drosophila via reducing the intracellular and mitochondrial reactive oxygen species (ROS) levels and inhibiting the caspase-3/caspase-9 activity. These results suggest that curcumin is a promising therapeutic compound for PD.
\end{abstract}

Keywords: Parkinson's Disease; Curcumin; Rotenone; Reactive Oxygen Species; Mitochondria; Drosophila

\section{INTRODUCTION}

Parkinson's disease (PD) is a progressive neurodegenerative disorder with high prevalence in aged people, affecting $2 \%$ of the population over 60 . The cardinal signs of PD are tremor, rigidity and bradykinesia, result-

\footnotetext{
*Author Contribution: Conceived and designed the experiments: WS ZL. Performed the experiments: ZL TL DY. Analyzed the data: ZL TL Wrote the paper: WS ZL.

"Present Address: Department of Human Anatomy, School of Medicine, Soochow University, Suzhou, China.
}

ed from a loss of dopaminergic neurons. The specific molecular mechanisms leading to neuronal death in PD are not fully understood yet $[1,2]$. Nevertheless, postmortem study implicates that oxidative damage and mitochondrial impairment could contribute to the cell death in PD [1]. Consistent with this finding, epidemiological studies also suggest that PD can be induced with mitochondrial complex I inhibitors such as the environmental toxins rotenone $[3,4]$.

Rotenone, a commonly used natural pesticide prepared from the roots of tropical plants, such as Derris elliptica, can freely cross cell and mitochondrial membranes. In vitro, rotenone has been shown to promote the accumulation and aggregation of alpha-synuclein and ubiquitin, cause oxidative damage, and endoplasmic reticulum stress, and lead to cell death $[5,6]$. In vivo, chronic exposure of rats to rotenone induces PD-like symptoms, including dopaminergic neurodegeneration and the occurrence of cytoplasmic inclusions similar to Lewy bodies [7]. Recent studies show that chronic exposure of Drosophila to rotenone recapitulates key features of Parkinsonism, including selective loss of dopaminergic neurons and locomotor deficits [8,9]. Although there are also study showing contradictory results [4], most of the evidences are consistent and suggesting that rotenone exposure contributes to PD-like symptom and that rotenone-based Parkinson's disease models can be used to test potential compounds for PD intervention.

Curcumin is a yellow curry spice derived from turmeric, which has been used as a food preservative and herbal medicine in India [10]. Curcumin is a potent antioxidant and anti-inflammatory compound [11]. Previous studies suggest that curcumin plays a protective role against amyloid beta toxicity in cell model of Alzheimer's disease and mouse models $[12,13]$. However, it is unknown whether curcumin can protect against rotenone toxicity in PD models. In this report, we used rotenonebased cell and Drosophila PD models to test the effects of curcumin. Our results showed that curcumin reduced rotenone-induced cell death in SH-SY5Y human neuro- 
blastoma cells via decreasing the intracellular and mitochondrial ROS levels and inhibiting caspase-3/caspase- 9 activity. Curcumin also rescued rotenone-induced locomotor impairment and early mortality and restrained dopaminergic neuronal degeneration in Drosophila via reducing mitochondrial ROS levels. These results suggest that curcumin may be a potential compound for PD intervention.

\section{METHODS}

\subsection{Materials}

Media and N2 supplements for cell culture were obtained from Invitrogen. 2',7'-dichlorofluorescein (DCF) diacetate (DCFDA) was obtained from Invitrogen. Rotenone and curcumin were from Sigma (St. Louis, MO, USA) were first dissolved in DMSO and then diluted into midia or fly food before use. The final concentration of DMSO was $0.2 \%$ in the media or fly food that were not alter cell and fly growth. The pan caspase inhibitor, z-VAD-fmk was purchased from Enzyme Systems Products (Livermore, CA, USA).

\subsection{Cell Culture and Measurement of Cell Death}

SH-SY5Y human neuroblastoma cells were grown in DMEM with 10\% Fetal boral serum (FBS) and antibiotic-antimycotic $(100 \mathrm{U} / \mathrm{ml}$ penicillin, $100 \mu \mathrm{g} / \mathrm{ml}$ streptomycin, and $2.5 \mu \mathrm{g} / \mathrm{ml}$ fungizone) solutions at $37^{\circ} \mathrm{C}$ in $5 \% \mathrm{CO} 2 / 95 \%$ air. For rotenone toxicity does-response studies, SH-SY5Y cells were added rotenone at $0-250$ $\mathrm{nM}$ concentrations.

To test effect of curcumin on rotenone toxicity, we used the LC 80 (the concentration causing $80 \%$ cell death) of rotenone. We pretreated cells with curcumin at $0,0.1,05,1$ and $5 \mu \mathrm{M}$ concentrations for $1 \mathrm{~h}$, and then exposed them to LC80 of rotenone for $24 \mathrm{~h}$. We also used a pan caspase inhibitor, z-VAD $(100 \mu \mathrm{M})$ as a positive control for curcumin protective experiments. Trypan blue exclusion was used to measure cell death by counting the number of dead (blue) and live cells in the cultures after rotenone exposure and/or curcumin treatment.

\subsection{Measurements of Intracellular ROS and Mitochondrial Superoxide}

The levels of cytosolic ROS were measured by redoxsensitive fluorophore DCFDA as previously described [14]. Briefly, cells were washed with PBS, and then incubated for $45 \mathrm{~min}$ with DCFDA, which is initially non-fluorescent and is converted by oxidation to the fluorescent molecular DCF. DCF was then quantified using a CytoFluor Multi-well Plate Reader, Series 400 (Perseptive Biosystems) with $485 \mathrm{~nm}$ excitation and 538 nm emission filters.

For detection of mitochondrial superoxide, MitoSOX Red and MitoTracker Green (Invitrogen) dissolved in DMSO were used at a final concentration of $5 \mu \mathrm{mol} / \mathrm{L}$ and $1 \mu \mathrm{mol} / \mathrm{L}$ respectively, according to the protocols of the manufacturers. Briefly, cells after rotenone exposure and/or curcumin treatment were loaded with the fluorogenic probe MitoSOX Red $(5 \mu \mathrm{mol} / \mathrm{L})$ for $20 \mathrm{~min}$. After removing MitoSOX Red and washing cells with Hanks' balanced salt solution, fluorescent images were captured by fluorescence microscope. MitoSOX Red is a fluorescent dye specific for the detection of $\mathrm{O} 2$ - in the mitochondria of live cells. To confirm the localization of MitoSOX Red to mitochondria, cells were also incubated with mitochondria marker (MitoTracker Green $(1 \mu \mathrm{mol} / \mathrm{L})$ for $20 \mathrm{~min}$ at the same time. The yellow fluorescence in the overlay images indicated that MitoSOX is inside of the mitochondria. Fluorescence pictures were taken by fluorescence microscopy with identical exposure settings. Ten random images from each well in a 24-well cell culture plate were taken for each parameter to obtain a mean value for statistical comparison. NIH Image J 5.0 software was used for the analysis of fluorescent intensity of MitoSOX in individual cell. Average intensity per cell was determined and more than 50 cells in each experimental group were analyzed. Each experiment was repeated three times.

\subsection{Measurement of Cellular Caspase Activity}

Caspase- 3 and caspase- 9 activities were measured by a colormetric method at A405 nm as previously described [14]. Cells were harvested in cell lysis buffer $(50 \mathrm{mM}$ HEPES, $1 \mathrm{mM}$ DTT, $0.1 \mathrm{mM}$ EDTA, 0.1\% CHAPS and $0.1 \%$ Triton $\mathrm{X}-100, \mathrm{pH} 7.4)$. DEVD-p-nitroanilide and LEHD-p-nitroanilide were the substrates for caspase-3 and caspase-9, respectively. The experiments were performed according to the manufacturer's protocol (Biosource International).

\subsection{The PD Model of Drosophila}

W1118 strain of Drosophila were grown on standard cornmeal medium at $25^{\circ} \mathrm{C}$ and fresh food media were changed every 3 days. To get PD model, the rotenone ( 0 - $125 \mathrm{nM}$ final concentration) were added freshly when the food changed.

\subsection{Fly Survival Curve}

Cohorts of 50 flies from each experimental group were monitored for survival. Flies were maintained on standard media, rotenone and rotenone with curcumin (0 - 5 $\mathrm{mM}$ final concentration) for untreated control, rotenone only and curcumin protect group. Mortality was scored 
daily and analyzed by using Kaplan-Meier survival curves. This experiment was repeated three times.

\subsection{Climbing Assay}

The fly locomotor ability was determined using a climbing assay (negative geotaxis assay) as described previously [15]. Cohorts of 60 flies from each genotype were subjected to the assay weekly. The tested flies were age-matched, randomly selected, anesthetized, and placed in a vertical plastic column (length, $25 \mathrm{~cm}$; diameter, $1.5 \mathrm{~cm}$ ). Flies were gently tapped to the bottom of the column. We counted and calculated the percentage of flies that could climb to, or above the median line of the cylinder in 10 seconds. Each week, the assay was repeated three times.

\subsection{Immunostaining and DA Neuron Counting}

Six neuronal DA clusters are normally present in each adult Drosophila brain hemisphere [15]. On the anterior side, there was a paired dopaminergic cluster, PAL (protocerebral anterolateral) and a paired cluster in the middle, PAM (paired anterolateral medial). On the posterior side, there were five clusters: PPM1 (unpaired), PPM2 (paired), PPM3 (paired) (protocerebral posterior medial), PPL1 and PPL2 (paired) (protocerebral posterolateral). These neurons express tyrosine hydroxylase (TH), which is an enzyme required for the biosynthesis of dopamine and often used as a marker for dopaminergic neurons. Thus, whole-mount brain immunostaining using specific anti-TH antibodies were performed to detect DA neurons as previously described [15]. Briefly, fluorescent immunostaining was performed on whole-mount dissected adult brain at $2-4$ weeks of age in the presence or absence of curcumin treatment and/or rotenone exposure. Cohorts of six to eight flies per experimental group were used for immunostaining. The dissected brains were mounted in Vectashield (Vector Laboratories). Rabbit polyclonal anti-TH (Chemicon) and mouse monoclonal anti-TH (Immunostar) were used as the primary antibodies. Alexa Fluor 488 goat anti-mouse IgG and Alexa Fluor 568 goat anti-mouse IgG (Invitrogen) were used as secondary antibodies. The numbers of DA neurons were scored in whole-mount brains under fluorescent (Zeiss LSM 250) and/or confocal microscopy (Zeiss LSM 510). We counted the TH-positive cells in all clusters except in the paired PAM cluster, because the density of the neurons in PAM was too high to allow precise quantification. Six brain sections were counted in each experimental group.

\subsection{Drosophila Brain Redox State Analysis}

The UAS-MTSroGFP2 was expressed in drosophila pan neurons by elav-Gal4 driver. The brain dissection and redox state analysis is performed as previously reported [16]. Briefly, the brains of elav-Gal4; UASMTSroGFP2 animals were dissected in PBS and placed in mounting medium (Vector, H-1000). RoGFP2 fluorescence was collected by using a $535 \mathrm{~nm}$ filter following excitation at $405 \mathrm{~nm}$ (Oxidized roGFP2) and $488 \mathrm{~nm}$ (Reduced roGFP2). The $20 \times$ lens and Z-scan by $10 \mathrm{um}$ stepsize was used to get the whole brain image. The Ratios of $405 \mathrm{~nm} / 488 \mathrm{~nm}$ fluorescences were counted by ImageJ software and data were analyzed using student $t$-test.

\subsection{Data Analysis}

Quantitative data were expressed as arithmetic means \pm SEM based on at least three separate experiments. Statistically significant differences among experimental groups were analyzed by analysis of variance (ANOVA) using Sigmastart 3.1 statistical soft ware (Aspire Software International, VA). A $P$ value $(<0.05)$ was considered significant.

\section{RESULTS}

\subsection{Curcumin Protected Against Rotenone Induced Apoptosis in SH-SY5Y Cells}

To address whether curcumin can restrain rotenoneinduced neurodegeneration, we exposed human dopaminergic neuroblastoma SH-SY5Y cells to various concentrations of rotenone for $24 \mathrm{~h}$. Rotenone (Figure 1(a)) caused apoptotic cell death in a dose-dependent manner (Figure 1(b)). The LC80 (the concentration causing 80\% cell death) of rotenone was $125 \mathrm{nM}$. To test the protective effect of curcumin (Figure 1(c)) on rotenone toxicity, we pretreated cells with curcumin for $1 \mathrm{~h}$, and then exposed them to $125 \mathrm{nM}$ rotenone for $24 \mathrm{~h}$. Curcumin protected against $125 \mathrm{nM}$ rotenone-induced cell death to a significant extent at a concentration of $100 \mathrm{~nm}$ and to a maximum extent at $1 \mu \mathrm{M}$ (Figure 1(d)).

\subsection{Rotenone Caused PD-Like Symptoms in Drosophila}

We generated a rotenone-based fly model using W1118 normal wild type Drosophila. We added rotenone in fly food at a concentration gradient of 5, 25, 75 and $125 \mu \mathrm{M}$ from day 1 post-eclosion and keep the same drug level in the food throughout the lifetime. We found that rotenone caused early death in a dose-dependent manner and is significant when compared with unexposed flies (Figure 2(a)). To measure the behavioral change resulted from rotenone exposure, we employed a climbing assay (negative geotactic test) at multiple time points in their lifetime. When flies were tapped to the 
(a)

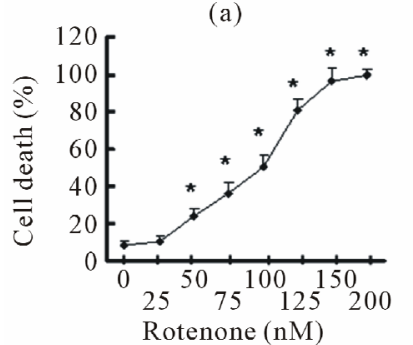

(c)

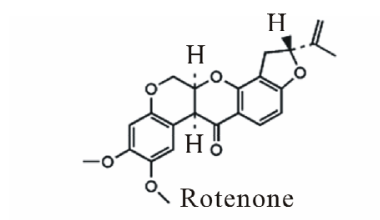

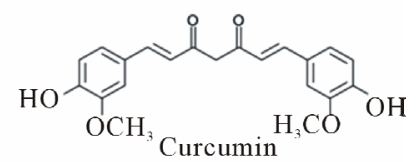

(b)

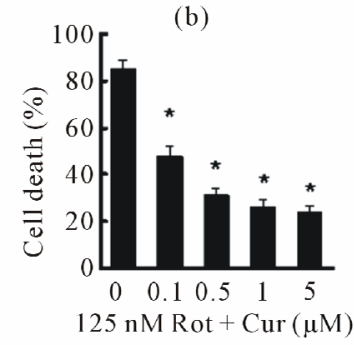

(d)
Figure 1. Curcumin protected against Rotenone caused cell death in vitro. (a) Chemical structure of Rotenone; (b) Dose response curve of Rotenone induced cell death in SH-SY5Y cells. SH-SY5Y cells were treated with Rotenone for $24 \mathrm{~h}$ in $2 \%$ FBS OPTI-1 media. Cell death was measured with Trypan blue assay. Values are expressed as means \pm SEM. Ratio of cell death in multiple groups were significantly higher than that of control group with no Rotenone exposure $\left({ }^{*} p<0.05\right)$; (c) Chemical structure of curcumin; (d) SH-SY5Y cells were pretreated with curcumin at the indicated concentrations for $1 \mathrm{~h}$, then either left untreated (control) or treated with $125 \mathrm{nM}$ rotenone for $24 \mathrm{~h}$. Cell death was measured with Trypan blue exclusion assay. Curcumin treated cells showed significant lower cell death rate when compared to those cells with rotenone exposure but no curcumin treatment $\left({ }^{*} p<0.05\right)$.

bottom of a vial, nearly all untreated control flies rapidly climbed to the top of the vial on day 7 and day 14. However, flies exposed to rotenone exhibited a significant dose-dependent motor deficit in performance, indicating locomotor dysfunction (Figure 2(b)). There was a progressive, dose dependent decline in climbing ability in flies with rotenone exposure. Rotenone at $75 \mu \mathrm{M}$ and 125 $\mu \mathrm{M}$ caused significant locomotor impairment and early mortality compared to the untreated group. Thus, we used these two concentrations to conduct further studies.

To assess whether rotenone induces degeneration in dopaminergic neurons, brains from flies with rotenone exposure were dissected and subjected to whole-mount brain immunostaining using specific anti-TH antibodies. We counted the TH-positive cellsin all the clusters (the details are described in the methods section) except the paired PAM cluster, be cause the density of the neurons in PAM was too high to allow precise quantification. In unexposed control flies, at 2 week of age, the DA clusters did not change significantly in number of TH-positive cells or their morphology (Figures 2(c) and (d)). However, at 2 week of age, flies exposed to rotenone showed a striking decrease of anti-TH-positive staining in all the DA clusters, indicating that rotenone induced a loss of DA neurons in the brain.

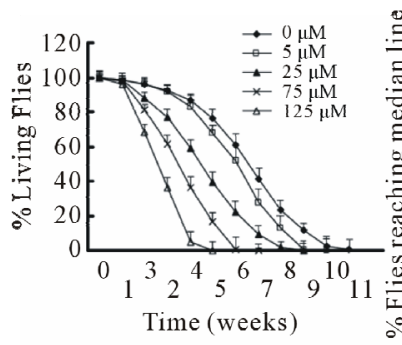

(a)
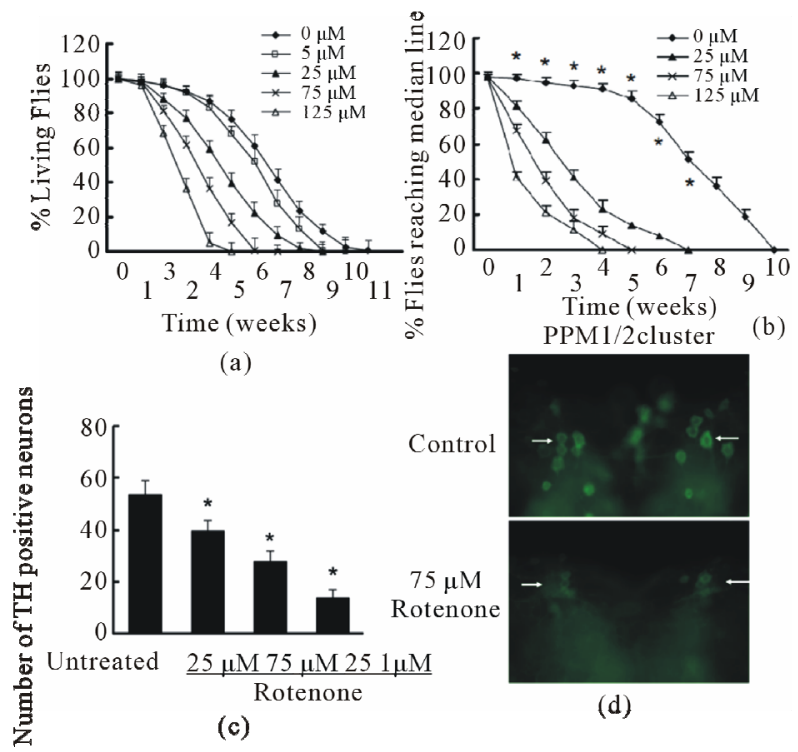

Figure 2. Rotenone induced PD-like phenotypes in Drosophila. $\mathrm{A}$ and $\mathrm{B}$, Rotenone at various concentration was added in fly food at 1 day post-eclosion through the lifetime. (a) Fly survival ratio were scored weekly. There were 60 flies in each group and 3 parallel experiments were carried out in total. Data were analyzed using Kaplan-Meier survival analysis (log-rank followed by Holm-Sidak method). Rotenone treatments at 25, 75 , and $125 \mathrm{nM}$ significantly reduced fly survival ratio compared with the group with no rotenone exposure $(p<0.05)$; (b) The locomotor activity was monitored weekly using climbing assays. There were 30 flies in each experimental group and this experiment was repeated three times in parallel. Rotenone treatment significantly reduced fly locomotor function compared with control with no rotenone exposure ${ }^{*} p<0.05$ analyzed with ANOVA); (c) and (d) Rotenone caused dopaminergic neuronal degeneration. Rotenone at 25, 75 and $125 \mu \mathrm{M}$ was added in fly food at 1 day post-eclosion for 14 days. The fly brains were dissected and subjected to whole mount brain immunoflouresence using anti-TH antibodies; (c) Quantification of anti-TH positive neurons in DA neuron clusters; (d) Representative images of PPM1/2 clusters of control and $75 \mu \mathrm{M}$ Rotenone group. Arrows indicate examples of TH-positive neurons in both conditions.

\subsection{Curcumin Ameliorated Rotenone-Induced PD-Like Symptoms in Drosophila}

To test the effect of curcumin in vivo, we added curcumin at $0,0.2,1,5,10 \mathrm{mM}$ in fly food from 1 day post-eclosion throughout the lifetime. The flies were also exposed to $75 \mu \mathrm{M}$ rotenone for the same time span. The fly survival and climbing activity were monitored weekly. $1 \mathrm{mM}$ curcumin significantly improved fly survival (Figure 3(a)) and prevented locomotor impairment induced by rotenone (Figure 3(b)). Increase of curcumin concentration in the fly food to more than $1 \mathrm{mM}$ did not further enhance its protective effect.

To further assess whether curcumin reduces dopaminergic neuron loss, flies exposed to $75 \mu \mathrm{M}$ rotenone 


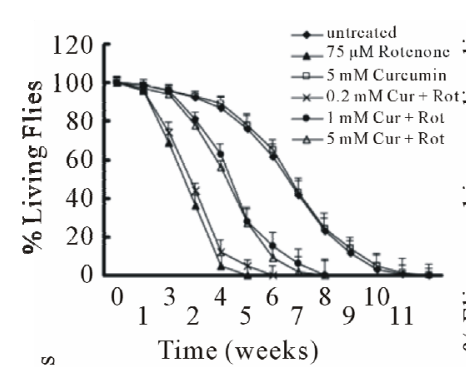

(a)

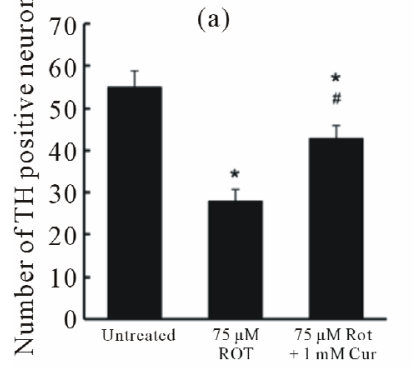

(c)

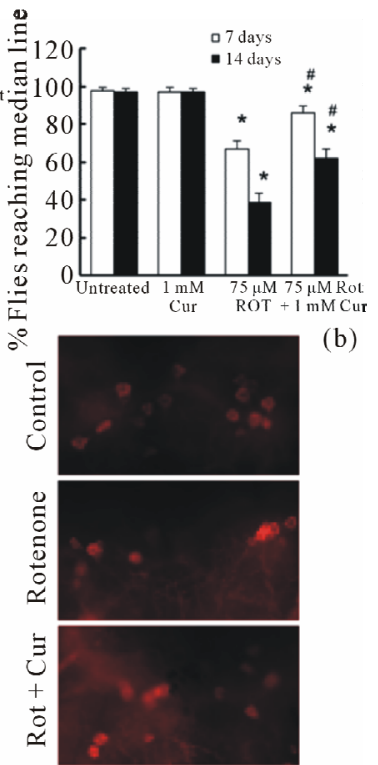

(d)

Figure 3. Curcumin attenuated rotenone-induced toxicity in drosophila. (a). Flies were exposed to $75 \mu \mathrm{M}$ Rotenone in the presence or absence of curcumin at various concentrations. Fly survival ratio was scored weekly. There were 60 flies in each group, and experiment was repeated 3 times in parallel. Survival data were analyzed by Kaplan-Meier log Rank survival analysis. Statistically significant differences were detected between no rotenone exposure and rotenone exposure group; between curcumin $(1$ and $5 \mathrm{mM})$ treated and non-treated flies that were exposed to rotenone $(p<0.05)$; (b). Flies were exposed with $75 \mu \mathrm{M}$ Rotenone in the presence or absence of 1 $\mathrm{mM}$ curcumin for 2 weeks. The locomotor function was monitored at 7 and 14 days using climbing assays. There were 30 flies in each experimental group. Data were analyzed by ANOVA. There were no significance between 7 day and 14 day assay in group untreated (control) and curcurmin alone ( ${ }^{*} p>$ 0.05 ). There were significant impairment in locomotor function both on 7 day and 14 day between groups with rotenone exposure ( no curcumin treatment) and control $\left({ }^{*} p<0.05\right)$. Flies exposed to rotenone and treated with curcumin showed a significant protection on locomotor function $\left({ }^{\#} p<0.05\right.$, compared to the group exposed to rotenone alone), but the toxicity of rotenone was not fully prevented $\left({ }^{*} p<0.05\right.$, compared to control); (c) and (d). Curcumin reduced Rotenone-induced loss of dopaminergic neurons. Flies were exposed with $75 \mu \mathrm{M}$ Rotenone in the presence or absence of $1 \mathrm{mM}$ curcumin for 2 weeks. The fly brains were dissected and subjected to whole mount brain immunoflouresence using anti-TH antibodies. (C). The total TH-positive neurons in dopaminergic neuron clusters (PAL, PPM1/2, PPM3, PPL1 and PPL2) were quantified in 3 different groups. The number of TH positive neurons in Curcumin alone group were not different from that in the control group (no rotenone and no curcumin, ${ }^{*} p>0.05$, data not shown). Rotenone exposed groups showed significant lower numbers of TH-positive neurons $\left({ }^{*} p<0.05\right.$, vs control, $n=6$ ) while curcumin treatment significantly restrained the toxicity of rotenone on TH-positive neurons $\left({ }^{\#} p<0.05\right.$, vs. Rotenone alone, $n=6$ ). D. Representative images of TH staining in PPM1/2 clusters of each experimental group.

were treated with $1 \mathrm{mM}$ curcumin. At 2 weeks of age, flies exposed to rotenone showed a striking decrease in anti-TH-positive staining in all DA clusters. However, curcumin significantly reduced rotenone-induced dopaminergic neuron loss in these flies (Figures 3(c), (d)).

\subsection{Curcumin Reduced Rotenone-Induced Intracellular and Mitochondrial ROS Levels in Vitro and in Vivo}

To further study the protective mechanism of curcumin, we measured intracellular ROS using the redoxsensitive fluorophore 2',7'-dichlorofluorescein (DCF) diacetate (DCFDA). Nonfluorescent DCFDA is converted by oxidation to the fluorescent molecule DCF. We found that the level of DCF in SH-SY5Y cells treated with $125 \mathrm{nM}$ rotenone was more than four folds higher than that in untreated cells (Figure 4(a)). However, this increase in DCF fluorescence was largely reversed by curcumin. To further explore whether curcumin alters mitochondrial ROS, we used MitoSOX, a specific dye for mitochondrial ROS. MitoSOX signal was localized in MitoTracker-stained organelles, indicating that the MitoSOX staining was specific to mitochondria. Mitochondrial ROS increased dramatically after $4 \mathrm{~h}$ exposure to rotenone (Figures 4(b), (c)). Pretreatment with curcumin for $1 \mathrm{~h}$ significantly reduced the rotenone-induced increase in mitochondrial ROS (Figures 4(b), (c)).

To test the antioxidant effect of curcumin in vivo, we employed a genetically encoded ratiometric redox sensor roGFP2 [16]. RoGFP2 is a ratiometric redox indicator, i.e. the ratios of fluorescence intensity from excitation wavelength $405 \mathrm{~nm}$ (Figure 5(a), oxidized state) to 488 $\mathrm{nm}$ (Figure 5(a), reduced state) can indicate the ROS level. This sensor allows us to detect real time ROS level in different tissues or cell types by using different GAL4 drivers in drosophila. The mitochondrial target sequence (MTS) were inserted upstream of roGFP2 that can specifically target roGFP2 to mitochondria. The pan neural driver elav-GAL4 was used to generate elav-GAL4; UAS-MTSroGFP2 flies and express roGFP2 in mitochondria of all the neurons. To observe the mitochondrial ROS level in neu rons, we dissected the whole brain of elav-GAL4; UAS-MTSroGFP2 flies on day 7 and day 14 after their exposure to $75 \mu \mathrm{M}$ rotenone with or without 1 $\mathrm{mM}$ curcumin in the presence. The exposure to rotenone caused an increase in ROS level compared to the vehicle control. When flies were rescued with $1 \mathrm{mM}$ curcumin, their ROS level was significantly reduced compared to that of flies only treated with rotenone (Figure 5(b)).

\subsection{Curcumin Inhibited Rotenone-Induced Caspase-3 and Caspase-9 Activation in SHSY5Y Cell}

Rotenone can activate caspase cascade to induce 


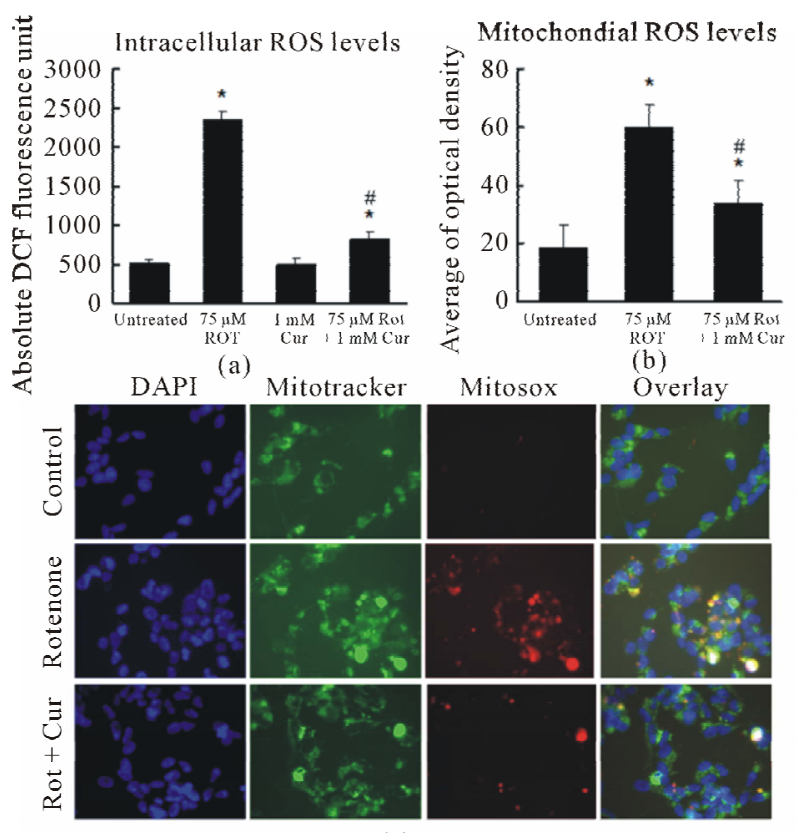

(c)

Figure. 4. Curcumin reduced intracellular and mitochondrial ROS levels. (a) Quantification of DCF fluorescence in SHSY5Y cells. Curcumin significantly reduced rotenone-induced intracellular ROS increase; (b) and (c) Curcumin significantly reduced rotenone-induced ROS in mitochondria. Digital photomicrograph under fluorescent illumination showing mitochondria superoxide signal is shown in (c) and the average optical density of MitoSOX is summarized in (b). The ROS level is indicated by the average value of cellular MitoSOX optical density in 10 random fields in each condition. Data are shown as the mean \pm SEM for three separate experiments. ${ }^{*} p<0.05$, ROS level was significantly different when compared with the cells with no rotenone and no curcumin treatment. ${ }^{*} p<0.05$, ROS level was significantly different when compared to cells with rotenone exposure).

apoptosis [5]. Consistent with precious findings, we found that $125 \mathrm{nM}$ rotenone increased both caspase-3 and caspase-9 activities (Figures 6(a) and (b)) in our mo- del. Caspase-3 and caspase-9 activity were in creased after exposure to rotenone for $2 \mathrm{~h}$ and reach peak after $12 \mathrm{~h}$. To examine whether curcumin alters rotenone-induced caspase- 3 and caspase- 9 activation, we pretreated the cells with $500 \mathrm{nM}$ curcumin for $1 \mathrm{~h}$, and followed $125 \mathrm{nM}$ rotenone exposures for $24 \mathrm{~h}$. We used a pan caspase inhibitor, $100 \mu \mathrm{M} z-\mathrm{VAD}$ as a positive control that can inhibit both caspase-3 and caspase- 9 activities and protect against cell death. Like z-VAD, curcumin significantly reduced rotenone-induced caspase- 3 and caspase-9 activation (Figure 6(c) and (d)).

\section{DISCUSSION}

The main finding of this study is that curcumin protects against rotenone induced toxicity in vitro (in SHSY5Y cells) and in vivo (in Drosophila). Curcumin
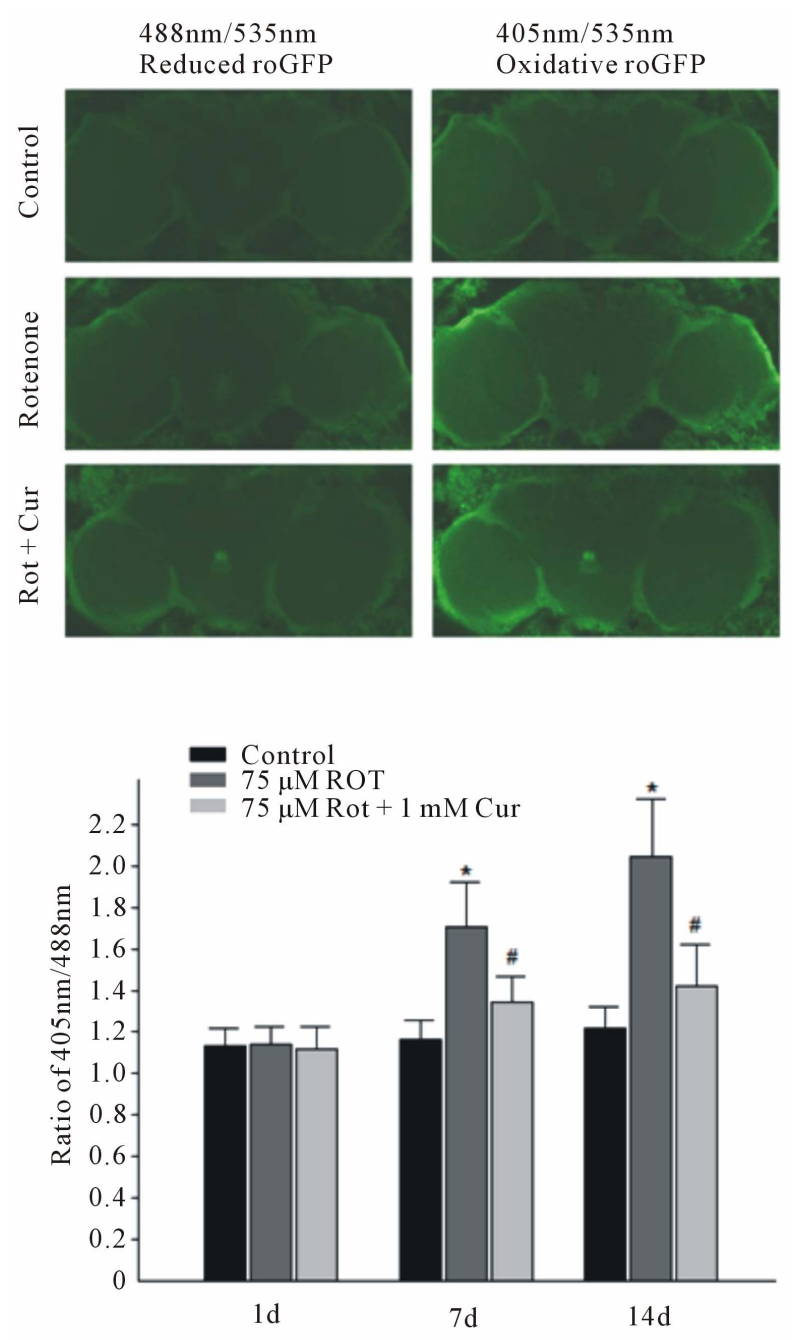

Figure. 5 Curcumin reduced mitochondrial ROS levels in drosophila neurons. (a) The image of oxidative roGFP2 in ElavGAL4; UAS-MTSroGFP2. From top to bottom: Control group, $75 \mu \mathrm{M}$ rotenone and $75 \mu \mathrm{M}$ rotenone plus $1 \mathrm{mM}$ curcumin; (b) Rotenone exposure induced increase of neural ROS signal at day 7 and day 14, while curcumin significantly reduced rotenone-induced ROS signal. Data are shown as mean \pm SEM. $\left({ }^{*} p\right.$ $<0.05$, vs cells with control. ${ }^{\#} p<0.05$, vs cells with rotenone exposure only).

attenuates rotenone-induced cell death in SH-SY5Y human neuroblastoma cells via reducing the intracellular and mitochondrial ROS levels and inhibiting caspase3/caspase-9 activity. Curcumin also can reduce the mitochondrial ROS level and increases survival time of fly in the presence of rotenone, alleviated their locomotor impairment and dopaminergic neuronal degeneration. These results suggest that curcumin may be a potential compound for PD intervention.

PD model that mimics the pathological changes in PD is very important in studying mechanisms underlying the etiogenesis of PD and also in searching for potential therapeutics for clinical use. Rotenone is used as a pesti- 

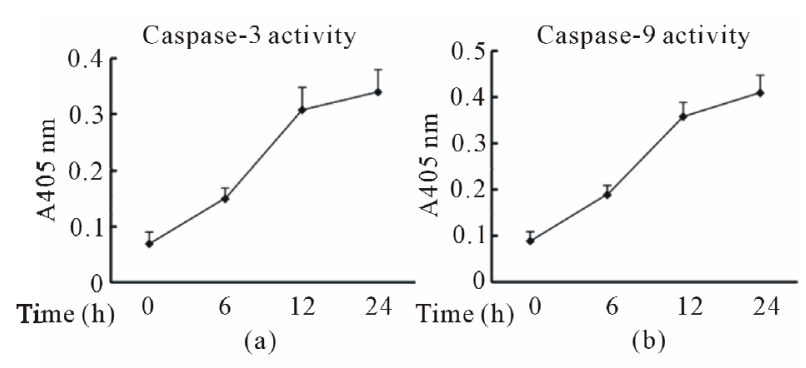

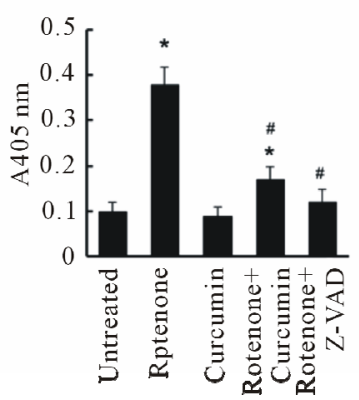

(c)

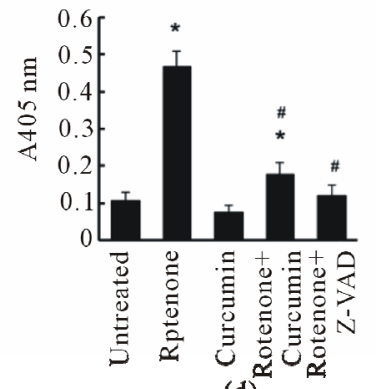

(d)
Figure 6. Curcumin inhibited Rotenone-induced caspase- 3 and caspase-9 activation in SHSY5Y cell. (a) Caspase-3 activity increased in a time-dependent manner after Rotenone exposure; (b) Caspase-9 activity increased in a time-dependent manner after Rotenone exposure; (c) Curcumin and Z-VAD (a pan caspase inhibitor, positive control) significantly reduced Rotenone-induced caspase-3 activation; (d) Curcumin and Z-VAD significantly attenuated Rotenone-induced caspase- 9 activation. Data are shown as mean \pm SEM of three separate experiments. $\left({ }^{*} p<0.05\right.$, results are significantly different when compared with control. ${ }^{\#} p<0.05$, results are significantly different when compared to cells with rotenone exposure only).

cide and it can inhibit mitochondrial complex I to present most pathological characteristics and clinical symptoms of PD. A number of rotenone-based toxin cell and rodent models have been used to study the molecular mechanisms of cell death and potential therapeutics for PD [5-8,17-20]. Here, we demonstrated that chronic exposure of Drosophila to rotenone recapitulated some key features of Parkinsonism, including early mortality, locomotor impairment and a selective loss of dopaminergic neurons. Increasing evidence from human and animal studies has suggested that oxidative damage critically contributes to neuronal loss in PD [21,22]. Oxidative stress increases with normal aging and neurodegenerative disorders [23-25]. Brains of PD patients have decreased levels of reduced glutathione (GSH), and there is oxidative damage to DNA, lipids and protein [21,26,27]. ROS responsible for this oxidative damage may be produced during dopamine metabolism or during oxidative phosphorylation [28,29]. Rotenone reduces complex I activity and increases ROS production [30,31]. Within complex I, upstream from the rotenone binding site, is a site of electron leak that can enhance ROS formation [32]. Our results showed that rotenone increases intra- cellular and mitochondrial ROS levels in the SH-SY5Y human neuroblastoma cells. The mitochondrial targeted roGFP2 is a good indicator for the redox state change in vivo drosophila neurodegeneration model by rotenone treatment. Using this indicator, we found that rotenone also increased the mitochondrial ROS level in drosophila. These results indicate that rotenone based cell and Drosophila are the good models to evaluate the therapeutic effect by candidate drug.

A growing body of evidences shows that inhibiting oxidative stress can prevent degeneration of nigrostriatal dopamine-containing neurons in animal models of neurodegenerative disorders. Curcumin is a potent antioxidant and anti-inflammatory compound. As a free radical scavenger, it is several times more potent than vitamin $\mathrm{E}$ [11]. It can protect the brain from lipid peroxidation [33], and scavenge NO-based radicals [34]. Curcumin has also been shown to play a protective role in amyloid beta peptide toxicity in AD models $[12,13]$. Recently, reports showed that curcumin also protects against PD-linked mutant alpha-synuclein and LRRK2-inudced neuron degeneration $[35,36]$. Consistent with these findings, our results demonstrate that curcumin reduces rotenone-induced death in SH-SY5Y cells. Using Rotenone-drosophila model, we found that curcumin dose-dependently attenuates rotenone-induced early mortality, locomotor impairment and dopaminergic neurodegeneration. Moreover, we found that curcumin significantly reduces rotenone-induced intracellular and mitochondrial ROS levels in SH-SY5Y cells and mitochondrial ROS level in drosophila. Furthermore, the caspase-3/caspase-9 activity is increased in rotenone-induced SH-SY5Y cells which is consistent with previous reports $[18,37]$. We also found the rotenone-induced increase of caspase-3/caspase- 9 activity is reduced by decreased ROS level in curcumin treatment. Taken together, these findings suggest that curcumin is a very promising therapeutic candidate and protects against neuron damages by both toxins and genetic mutations. Caspase- 9 activities and protect against cell death. The fact that curcumin reduces the ROS levels providing a molecular basis underlying neuroprotection against rotenone toxicity and may be a potential candidate for PD intervention. Curcumin can penetrate the blood brain barrier, is relatively broad therapeutic index $[12,13,38,39]$. In clinical trials with curcumin, cancer patients have not shown adverse effects with doses from 2000 to $8000 \mathrm{mg} /$ day [40]. Treatment with curcumin of 160 - $5000 \mathrm{ppm}$ reduces oxidative damage and amyloid pathology in an $\mathrm{AD}$ transgenic mouse [13]. Taken together, these studies indicate that curcumin is relatively safe for animals and humans and is a potentially attracttive therapeutic drug for PD and other neurodegenerative diseases. However, there is evidence that curcumin is poorly absorbed and heavily metabolized in animals 
which suggests the need of using large dose $(1 \mathrm{mM})$ to achieve neuroprotective effect in flies. The development of curcumin derivatives with higher bioavailability may lead to the better therapeutic utility of curcumin for PD intervention.

\section{CONCLUSION}

The Rotenone based cell and Drosophila models can provide a valuable system for preclinical investigation on PD therapeutics. We demonstrate that curcumin can protect against Rotenone-induced neuronal toxicity in vitro and in vivo via reducing ROS level and inhibiting capase-3/-9 activity. To our knowledge, this is the first report to show that curcumin has a protective effect in Rotenone-based PD models, suggesting that curcumin can be a promising therapeutic compound for PD.

\section{REFERENCES}

[1] Dawson, T.M., Dawson, V.L. (2003) Molecular pathways of neurodegeneration in Parkinson's disease. Science, 302, 819-822. doi: $10.1126 /$ science. 1087753

[2] Ross, C.A., Smith, W.W. (2007) Gene-environment interactions in Parkinson's disease. Parkinsonism \& Related Disorders, 13, S309-S315. doi:10.1016/S1353-8020(08)70022-1

[3] Dick, F.D. (2006) Parkinson's disease and pesticide exposures. British Medical Bulletin, 79-80, 219-231. doi:10.1093/bmb/ldl018

[4] Hoglinger, G.U., Oertel, W.H. and Hirsch, E.C. (2006) The rotenone model of parkinsonism-The five years inspection. Journal of Neural Transmission, 70, 269-272. doi:10.1007/978-3-211-45295-0 41

[5] Sherer, T.B., Betarbet, R., Stout, A.K., Lund, S., Baptista, M., Panov, A.V., Cookson, M.R. and Greenamyre, J.T. (2002) An in vitro model of Parkinson's disease: Linking mitochondrial impairment to altered alpha-synuclein metabolism and oxidative damage. The Journal of Neuroscience, 22, 7006-7015.

[6] Betarbet, R., Canet-Aviles, R.M., Sherer, T.B., Mastroberardino, P.G., McLendon, C., Kim, J.H., Lund, S., Na, H.M., Taylor, G. and Bence, N.F. (2006) Intersecting pathways to neurodegeneration in Parkinson's disease: Effects of the pesticide rotenone on DJ-1, alpha-synuclein, and the ubiquitin-proteasome system. Neurobiology of Disease, 22, 404-420. doi:10.1016/j.nbd.2005.12.003

[7] Betarbet, R., Sherer, T.B., MacKenzie, G., Garcia-Osuna, M., Panov, A.V. and Greenamyre, J.T. (2000) Chronic systemic pesticide exposure reproduces features of Parkinson's disease. Nature Neuroscience, 3, 1301-1306. doi: $10.1038 / 81834$

[8] Coulom, H. and Birman, S. (2004) Chronic exposure to ro- tenone models sporadic Parkinson's disease in Drosophila melanogaster. The Journal of Neuroscience, 24, 10993-10998. doi:10.1523/JNEUROSCI.2993-04.2004

[9] Hosamani, R., Ramesh, S.R. and Muralidhara. (2010)
Attenuation of rotenone-induced mitochondrial oxidative damage and neurotoxicty in Drosophila melanogaster supplemented with creatine. Neurochemical Research, 35, 1402-1412. doi:10.1007/s11064-010-0198-Z

[10] Kelloff, G.J., Crowell, J.A., Steele, V.E., Lubet, R.A., Malone, W.A., Boone, C.W., Kopelovich, L., Hawk, E.T., Lieberman, R. and Lawrence, J.A. (2000) Progress in cancer chemoprevention: development of diet-derived chemopreventive agents. The Journal of Nutrition, $\mathbf{1 3 0}$, 467S-471S.

[11] Zhao, B.L., Li, X.J., He, R.G., Cheng, S.J. and Xin, W.J. (1989) Scavenging effect of extracts of green tea and natural antioxidants on active oxygen radicals. Cell Biophysics, 14, 175-185.

[12] Yang, F., Lim, G.P., Begum, A.N., Ubeda, O.J., Simmons, M.R., Ambegaokar, S.S., Chen, P.P., Kayed, R., Glabe, C.G. and Frautschy, S.A. (2005) Curcumin inhibits formation of amyloid beta oligomers and fibrils, binds plaques, and reduces amyloid in vivo. The Journal of Biological Chemistry, 280, 5892-5901. doi:10.1074/jbc.M404751200

[13] Lim, G.P., Chu, T., Yang, F., Beech, W., Frautschy, S.A. and Cole, G.M. (2001) The curry spice curcumin reduces oxidative damage and amyloid pathology in an Alzheimer transgenic mouse. The Journal of Neuroscience, 21, 83708377.

[14] Smith, W.W., Jiang, H., Pei, Z., Tanaka, Y., Morita, H., Sawa, A., Dawson, V.L., Dawson, T.M. and Ross, C.A. (2005) Endoplasmic reticulum stress and mitochondrial cell death pathways mediate A53T mutant alpha-synuclein-induced toxicity. Human Molecular Genetics, 14, 3801-3811. doi:10.1093/hmg/ddi396

[15] Liu, Z., Wang, X., Yu, Y., Li, X., Wang, T., Jiang, H., Ren, Q., Jiao, Y., Sawa, A., Moran, T. and Smith, W.W. (2008) A Drosophila model for LRRK2-linked parkinsonism. Proceedings of the National Academy of Sciences of United States of America, 105, 2693-2698. doi:10.1073/pnas.0708452105

[16] Liu, Z., Celotto, A.M., Romero, G., Wipf, P. and Palladino, M.J. (2011) Genetically encoded redox sensor identifies the role of ROS in degenerative and mitochondrial disease pathogenesis. Neurobiology of Disease, 45, 362-368. doi:10.1016/i.nbd.2011.08.022

[17] Ahmadi, F.A., Linseman, D.A., Grammatopoulos, T.N., Jones, S.M., Bouchard, R.J., Freed, C.R., Heidenreich, K.A. and Zawada, W.M. (2003) The pesticide rotenone induces caspase-3-mediated apoptosis in ventral mesencephalic dopaminergic neurons. Journal of Neurochemistry, 87, 914-921. doi:10.1046/j.1471-4159.2003.02068.x

[18] Newhouse, K., Hsuan, S.L., Chang, S.H., Cai, B., Wang, Y. and Xia, Z. (2004) Rotenone-induced apoptosis is mediated by $\mathrm{p} 38$ and JNK MAP kinases in human dopaminergic SH-SY5Y cells. Society of Toxicology, 79, 137146. doi:10.1093/toxsci/kfh089

[19] Ramachandiran, S., Hansen, J.M., Jones, D.P., Richardson, J.R. and Miller, G.W. (2007) Divergent mechanisms of paraquat, $\mathrm{MPP}^{+}$, and rotenone toxicity: Oxidation of thioredoxin and caspase- 3 activation. Society of Toxicology, 95, 163-171. doi:10.1093/toxsci/kfl125 
[20] Leung, K.W., Yung, K.K., Mak, N.K., Chan, Y.S., Fan, T.P. and Wong, R.N. (2007) Neuroprotective effects of ginsenoside-Rg1 in primary nigral neurons against rotenone toxicity. Neuropharmacology, 52, 827-835. doi:10.1016/j.neuropharm.2006.10.001

[21] Dexter, D.T., Carter, C.J., Wells, F.R., Javoy-Agid, F., Agid, Y., Lees, A., Jenner, P. and Marsden, C.D. (1989) Basal lipid peroxidation in substantia nigra is increased in Parkinson's disease. Journal of Neurochemistry, 52, 381389. doi:10.1111/j.1471-4159.1989.tb09133.x

[22] Sian, J., Dexter, D.T., Lees, A.J., Daniel, S., Agid, Y., Javoy-Agid, F., Jenner, P. and Marsden, C.D. (1994) Alterations in glutathione levels in Parkinson's disease and other neurodegenerative disorders affecting basal ganglia. Annals of Neurology, 36, 348-355. doi:10.1002/ana.410360305

[23] Giasson, B.I., Duda, J.E., Murray, I.V., Chen, Q., Souza, J.M., Hurtig, H.I., Ischiropoulos, H., Trojanowski, J.Q. and Lee, V.M. (2000) Oxidative damage linked to neurodegeneration by selective alpha-synuclein nitration in synucleinopathy lesions. Science, 290, 985-989. doi:10.1126/science.290.5493.985

[24] Tabner, B.J., Turnbull, S., El-Agnaf, O.M. and Allsop, D. (2002) Formation of hydrogen peroxide and hydroxyl radicals from $\mathrm{A}$ (beta) and alpha-synuclein as a possible mechanism of cell death in Alzheimer's disease and Parkinson's disease. Free Radical Biology and Medicine 32, 1076-1083. doi:10.1016/S0891-5849(02)00801-8

[25] Maguire-Zeiss, K.A., Short, D.W. and Federoff, H.J. (2005) Synuclein, dopamine and oxidative stress: Co-conspirators in Parkinson's disease? Molecular Brain Research, 134, 18-23. doi:10.1016/j.molbrainres.2004.09.014

[26] Alam, Z.I., Jenner, A., Daniel, S.E., Lees, A.J., Cairns, N., Marsden, C.D., Jenner, P. and Halliwell, B. (1997) Oxidative DNA damage in the parkinsonian brain: An apparent selective increase in 8-hydroxyguanine levels in substantia nigra. Journal of Neurochemistry, 69, 1196-1203. doi:10.1046/j.1471-4159.1997.69031196.x

[27] Alam, Z.I., Daniel, S.E., Lees, A.J., Marsden, D.C., Jenner, P. and Halliwell, B. (1997) A generalised increase in protein carbonyls in the brain in Parkinson's but not incidental Lewy body disease. Journal of Neurochemistry, 69, 1326- 1329. doi:10.1046/j.1471-4159.1997.69031326.x

[28] Hasegawa, E., Takeshige, K., Oishi, T., Murai, Y. and Minakami, S. (1990) 1-Methyl-4-phenylpyridinium (MPP ${ }^{+}$ induces NADH-dependent superoxide formation and enhances NADH-dependent lipid peroxidation in bovine heart submitochondrial particles. Biochemical and Biophysical Research Communications, 170, 1049-1055. doi:10.1016/0006-291X(90)90498-C

[29] Lotharius, J. and O'Malley, K.L. (2000) The parkinsonism-inducing drug 1-methyl-4-phenylpyridinium triggers intracellular dopamine oxidation. A novel mechanism of toxicity. The Journal of Biological Chemistry, 275, 3858138588. doi:10.1074/jbc.M005385200
[30] Cassarino, D.S., Fall, C.P., Swerdlow, R.H., Smith, T.S., Halvorsen, E.M., Miller, S.W., Parks, J.P., Parker, W.D., Jr. and Bennett, J.P., Jr. (1997) Elevated reactive oxygen species and antioxidant enzyme activities in animal and cellular models of Parkinson's disease. Biochimica et Biophysica Acta, 1362, 77-86. doi:10.1016/S0925-4439(97)00070-7

[31] Votyakova, T.V. and Reynolds, I.J. (2001) DeltaPsi(m)Dependent and -independent production of reactive oxygen species by rat brain mitochondria. Journal of Neurochemistry, 79, 266-277. doi:10.1046/j.1471-4159.2001.00548.x

[32] Hensley, K., Pye, Q.N., Maidt, M.L., Stewart, C.A., Robinson, K.A., Jaffrey, F. and Floyd, R.A. (1998) Interaction of alpha-phenyl-N-tert-butyl nitrone and alternative electron acceptors with complex I indicates a substrate reduction site upstream from the rotenone binding site. Journal of Neurochemistry, 71, 2549-2557. doi:10.1046/j.1471-4159.1998.71062549.x

[33] Martin-Aragon, S., Benedi, J.M. and Villar, A.M. (1997) Modifications on antioxidant capacity and lipid peroxidation in mice under fraxetin treatment. Journal of Pharmacy and Pharmacology, 49, 49-52. doi:10.1111/j.2042-7158.1997.tb06751.x

[34] Sreejayan and Rao, M.N. (1997) Nitric oxide scavenging by curcuminoids. Journal of Pharmacy and Pharmacology, 49, 105-107. doi:10.1111/j.2042-7158.1997.tb06761.X

[35] Liu, Z., Yu, Y., Li, X., Ross, C.A. and Smith, W.W. (2011) Curcumin protects against A53T alpha-synuclein-induced toxicity in a PC12 inducible cell model for Parkinsonism. Pharmacological Research, 63, 439-444. doi:10.1016/i.phrs.2011.01.004

[36] Yang, D., Li, T., Liu, Z., Arbez, N., Yan, J., Moran, T.H., Ross, C.A. and Smith, W.W. (2012) LRRK2 kinase activity mediates toxic interactions between genetic mutation and oxidative stress in a Drosophila model: Suppression by curcumin. Neurobiology of Disease, 47, 385-392. doi:10.1016/j.nbd.2012.05.020

[37] Wang, X., Qin, Z.H., Leng, Y., Wang, Y., Jin, X., Chase, T.N. and Bennett, M.C. (2002) Prostaglandin A1 inhibits rotenone-induced apoptosis in SH-SY5Y cells. Journal of Neurochemistry, 83, 1094-1102. doi:10.1046/j.1471-4159.2002.01224.x

[38] Chainani-Wu, N. (2003) Safety and anti-inflammatory activity of curcumin: A component of tumeric (Curcuma longa). Journal of Alternative and Complementary Medicine, 9, 161-168. doi:10.1089/107555303321223035

[39] Sharm,a R.A., Euden, S.A., Platton, S.L., Cooke, D.N., Shafayat, A., Hewitt, H.R., Marczylo, T.H., Morgan, B., Hemingway, D. and Plummer, S.M. (2004) Phase I clinical trial of oral curcumin: Biomarkers of systemic activity and compliance. Clinical Cancer Research, 10, 68476854.

[40] Hsu, C.H. and Cheng, A.L. (2007) Clinical studies with curcumin. Advances in Experimental Medicine and Biology, 595, 471-480. doi:10.1007/978-0-387-46401-5_21 


\section{Abbreviations}

PD, Parkinson's Disease; TH, tyrosine hydroxylase; DA, dopaminergic; ROS, reactive oxygen species; DMEM, Dulbecco's Modified Eagle Medium; DCFDA, 2',7'-dichlorodihydrofluorescein diacetate; DMSO, Dimethyl sulfoxide; LC 80 , the concentration causing $80 \%$ cell death; ANOVA, an analysis of variance. 